\title{
Guidelines to Transform Industry Clusters in Virtual Organization Breeding Environments - A Case Study
}

\author{
Fabiano Baldo ${ }^{1}$ and Ricardo J. Rabelo ${ }^{2}$ \\ ${ }^{1}$ Department of Computer Science, Santa Catarina State University, \\ Zip Code 89223-100, Joinville, Brazil \\ ${ }^{2}$ Department of Automation and Systems, Federal University of Santa Catarina, \\ PO Box 476, Zip Code 88040-970, Florianópolis, Brazil \\ baldo@joinville.udesc.br, rabelo@das.ufsc.br
}

\begin{abstract}
This paper encompasses the specific problem of how transforming industry clusters in environments prepared to create Virtual Organizations. This kind of coalitions is known as Virtual Organization Breeding Environments (VBEs). In order to reach the envisaged objective a set of guidelines for implementing VBEs has been proposed and its current results have been presented here. These guidelines are strongly based on modeling frameworks. This approach is being tested in a cluster of moulds and dies producers from Brazil. In the future, it is intended to use these guidelines as a structured approach to systematize the implementation of any type of VBE.
\end{abstract}

Keywords: Virtual Organization, Virtual Organization Breeding Environment, Members Preparedness, VBE Implementation Methodology.

\section{Introduction}

Recently, Brazilian Small and Medium size Enterprises (SME) are increasingly establishing alliances with local competitor in order to increase their national and international competitiveness. The importance of strategic alliances has been pointed out by many authors as a way to augment industries' competitiveness [1]. Pursuing the advantages brought by strategic alliances, in 1990's Brazilian industry clusters started to create strategic alliances over the country based on a so-called local productive arrangement concept. Despite of the benefits of this initiative, it has been realized that this form of alliance is limited to face some new business requirements, as volatility of economics, commercial barriers, global competition and innovation needs. [2]. New forms of alliances have been recently arisen to cope with these requirements under the scope of the conceptualization called Collaborative Networked Organizations (CNO).

Considering the original goals of local productive arrangements, among the new collaboration forms identified by Camarinha-Matos et al. [3], VBE has been seen as its natural evolution. Virtual Organization Breeding Environments (VBEs) can be defined as a long-term alliance of organizations aimed at offering conditions to 
support the rapid configuration of virtual organizations (VO) to attend given business opportunities [4]. Among many competitive advantages, VBEs have the potential to enhance collaboration and knowledge sharing among members, to decrease costs and risks, to leave them more competitive acting jointly and to reach new markets.

The establishment of a VBE is a very complex task regarding the number of technical and non-technical aspects to embrace. Existing VBEs have fundamentally been created by ad-hoc approaches, which have created some obstacles for their proper scalability and configurability during their typical evolution [5] [6]. In the last years, a relevant number of works have presented more solid theoretical models for $\mathrm{CNO}$ formation, including VBE creation. An important contribution to answer this question has been proposed by Romero et al. [7]. In this work it has been devised a methodology to create VBEs based on a reference framework for modeling CNOs. Although presenting more concrete sequence of actions to create VBEs, it is too generic yet to be applied indeed. Therefore, it requires an additional effort when trying to build VBEs using it. This additional effort is mainly required to harmonize the considerable discrepancy of preparedness among VBE members candidate. Afsarmanesh et al. [4] state that candidate members' preparedness is a crucial element for the successful creation of VBEs. As can be seen, it is not sufficient to have methodologies to implement VBE if these methodologies do not take into consideration the VBE candidates' preparedness. So, the problem is how to take into account candidate's preparedness when specifying the VBE implementation methodology?

In order to contribute to answer this question, it is assumed as hypothesis that a more correct identification of candidates' preparedness would speed-up the VBE creation process and decrease launching costs, as well as increase members' confidence and their overall preparation. In this sense, this paper presents a set of guidelines to transform industry clusters in VBEs where the candidates' preparedness has the central hole in the specification of the VBE implementation methodology. The contributions of this work are been tested in a case study performed in a cluster of moulds and dies producers from Brazil.

In this paper, section 2 presents the guidelines proposed to implement VBEs. Section 3 describes the VBE model specified for the case study. Section 4 depicts the characteristics to assess VBE member candidates. Section 5 shows the evaluation of the VBE member candidates. Section 6 specifies the VBE implementation methodology. Finally, section 7 presents some conclusions and future work.

\section{Guidelines for VBE Implementation}

This section presents the set of guidelines for implementing VBEs. This approach has been developed based on previous modeling frameworks and implementation methodologies that will be presented in section 3. The structure approach is composed of the following steps presented in Figure 1:

1. VBE Model Specification. This step aim at specifying a model to represent every key-element to be considered for implementing a VBE for a particular cluster. This specification should be based on reference frameworks, so that, a complete reviewing of the state-of-the-art is needed. This representation provides a better understanding of all elements involved in the VBE implementation. 


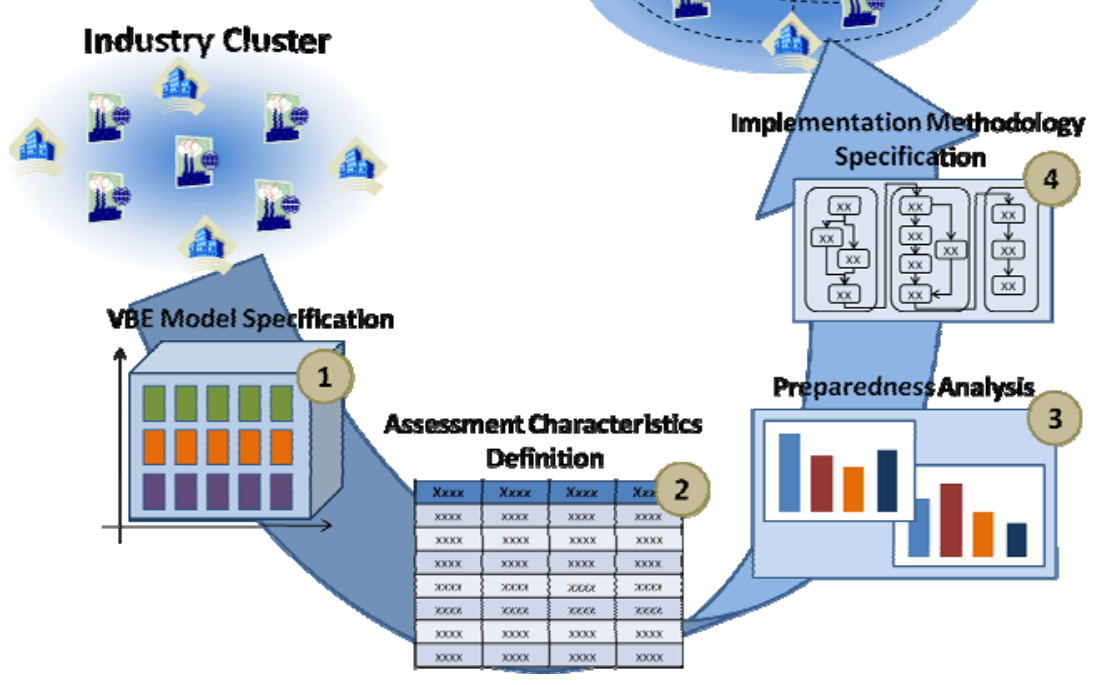

Fig. 1. Steps for VBE Implementation

2. Assessment Characteristics Definition. This step defines the assessment characteristics that are going to be used to check cluster members' preparedness. Based on the model previously specified, this step intends to describe the relevant characteristics that should be considered when assessing actual organizations' preparedness to be part of a VBE. These characteristics should be classified according to some predefined perspectives.

3. Preparedness analysis. This step aims at applying individual assessment in order to map the main weakness and strengths of each organization and thus to have a more comprehensive and precise overview about the global organizations' needs. This assessment can be performed using questionnaires specified based on assessment characteristics defined in step2 and its main propose is to provide a more tuned plan to implement the methodology for the VBE implementation.

4. Implementation Methodology Specification. This step specifies the methodology for implementing the VBE for the specific group of organizations. This methodology identifies the activities, their relationships and the involved actors involved in the VBE implementation. This methodology is specified based on the organizations' preparedness analysis done in step 3.

Next sections detail these steps applied in a case study performed in a cluster of moulds and dies producers called NuFerJ that is located in Joinville - Brazil. 


\section{VBE Model Specification}

In order to better understand the whole set of characteristics involved in the construction of a VBE it is necessary to model this VBE using reference frameworks. Several works have addressed the problem related to model CNOs. A comprehensive review of the state-of-the-art about such subject is found in Baldo and Rabelo [8]. In this work, the authors state that among the reviewed CNO modeling frameworks, ARCON is the most appropriated one to model VBEs for industry clusters. This assumption is

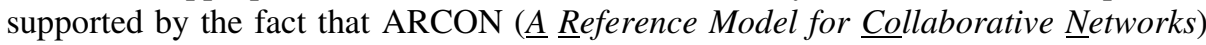
brings the possibility to model generic and abstract representations for understanding the involved CNO's entities and the relationship among them [9]. So, ARCON intends to be used as the basis for deriving models for any manifestations of CNOs. In very general terms, this is made applying three inter-related perspectives: Life Cycle; Environment Characteristics; and Model Intents.

Having selected the framework used to model the VBE for a specific cluster, it is necessary to collect significant data to model the specific VBE. For the NuFerJ particular case, it has been used information collected mainly from the cluster itself and from an educational institution that has performed some studies inside NuFerJ. Concerning NuFerJ characterization, it can be said that NuFerJ (Group of Moulds and Dies Industries of Joinville) is a cluster founded in 1993 that has about 50 members. Several members are competitors with each other and their main customers are automobile and household appliance companies. Moulds and dies are very unique parts that are produced only once and each one of them uses to be very complex to manufacture. This kind of industry uses high-speed CNC machines to transform large blocks of steel in complex moulds [10]. Considering the increasing and extreme hard world-wide competition, NuFerJ is looking for an alternative model which allows its members to better and more effectively prepare themselves for this new reality.

Taking into account ARCON modeling framework, a NuFerJ VBE Model has been created. This model classifies every element necessary to design the NuFerJ VBE, which in turn will guide the work towards its implementation. Baldo and Rabelo [8] presents the VBE model created for NuFerJ, considering all the characteristics and elements involved in the Endogenous and Exogenous ARCON's subspaces [11].

\section{Assessment Characteristics Definition}

As mention before, to implement a VBE it is necessary first to know quite well the cluster and its members in order to specify the implementation methodology. A way to perform such recognition is doing an assessment of the cluster members. This assessment evaluates the cluster members' preparedness to be part of a VBE. Conceive assessment strategies, e.g. questionnaires, base of the information available at the VBE model is to complex due to its broad coverage. So, to overcome this obstacle it is proposed to extract from the model the relevant characteristics and to organize them into perspectives of assessment. Table 1 shows the assessment characteristics extracted from the NuFerJ VBE model and used to assess NuFerJ members' preparedness. 


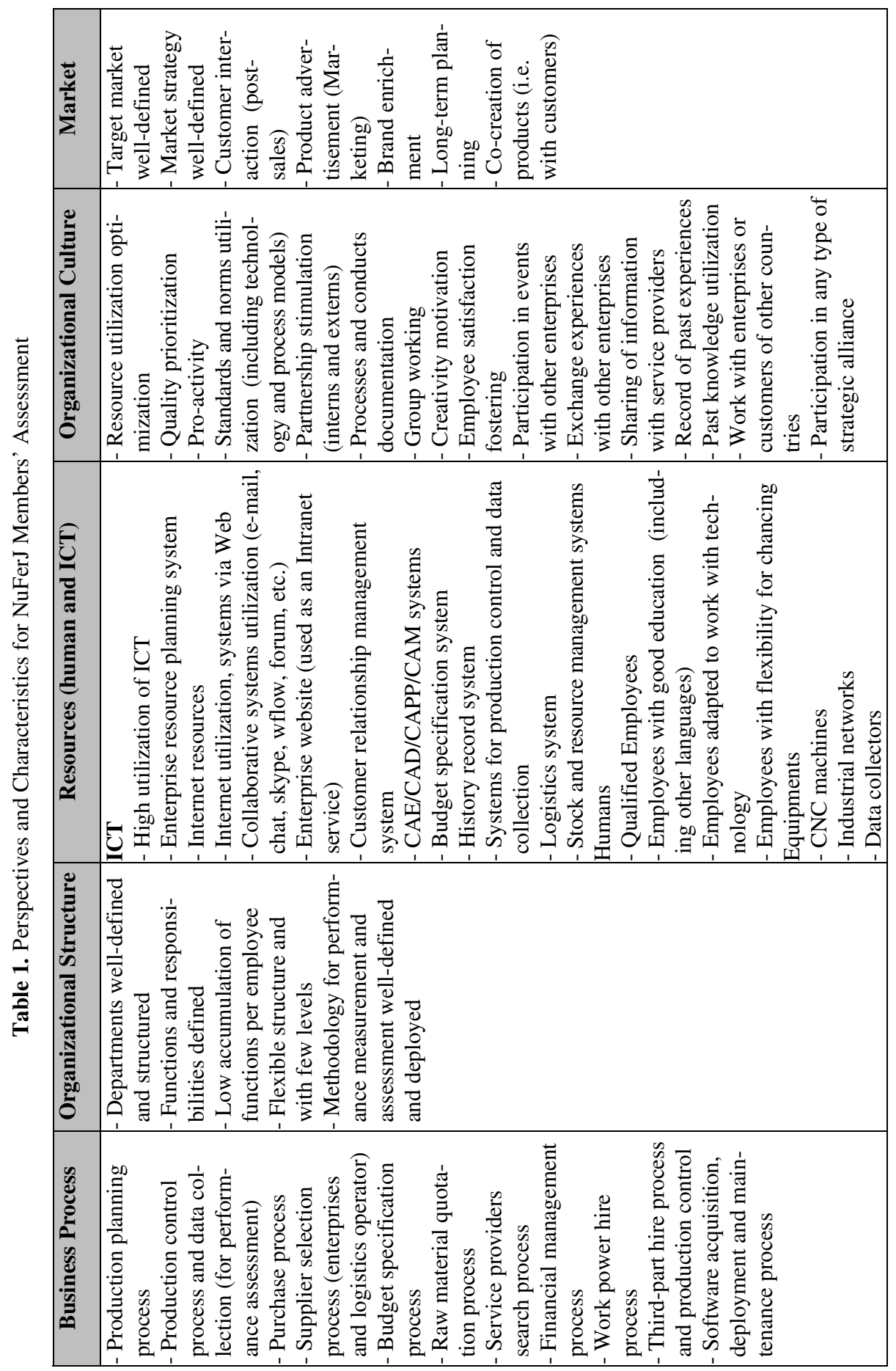




\section{Preparedness Analysis}

The characteristics contained in the perspectives presented in section 4 can be used to specify questions for evaluating how prepared an industry is to be part of a VBE. Roughly, it can be said that each question is based on a characteristic presented in Table 1. It means, each characteristic in Table 1 has been written as a question in order to ask for each candidate if it presents such VBE requirement. However, when appropriated, some characteristics have been combined into a single question due to their intrinsic correlation. Figure 2 presents an example of question.

1. Does the organization have a defined production planning process?
$\square$ Yes $\square$ Excellent $\square$ Good $\square$ Not good $\square$ Bad $\square$ No
Comments:

Fig. 2. Example of question presented in the questionnaire

The questionnaire conceived contains 45 questions divided into five perspectives as follows:

- Business Process: 9 questions;

- Organizational Structure: 4 questions;

- Resources (human and ICT): 14 questions;

- Organizational Culture: 13 questions;

- Market: 5 questions.

Each question has been conceived in a way to estimate the level of preparation of an industry. This level is estimated through a scale of values on which the industries should fit in one of them. This scale is composed of the following values: excellent, good, not good and bad. Besides that, each question has two check boxes containing yes and no values, respectively. These options are necessaries in situations where a question is not applied for a specific industry or the industry does not have any manifestation of such characteristic stated in the question.

Having the questionnaire prepared it is time to start the interviews. The questionnaire has been applied to six industries that participating in the NuFerJ cluster. The choice of those six has been aided by a manager that knows pretty well most of the industries and it has been chosen medium size industries with high organizational degree and small size industries with low organizational degree.

The results obtained through the interviews have been first charted and afterwards analyzed and finally graphically plotted. In order to chart the obtained data the scale presented in the questionnaire has been converted as follows: "excellent" $\rightarrow 4$; "good" $\rightarrow 3$; "not good" $\rightarrow 2$; "bad" $\rightarrow 1$; "no" $\rightarrow 0$.

Figure 3 shows an overview about the industries' preparedness. Considering the business process perspective, in general terms the industries are not much prepared, as it can be seen in Figure 3 where the average has reached 2.28. It means that they do not have a good systematization of daily performed business processes. This interferes negatively to reach industries' common business processes definition. Concerning the organizational structure the industries have reached a better level of 


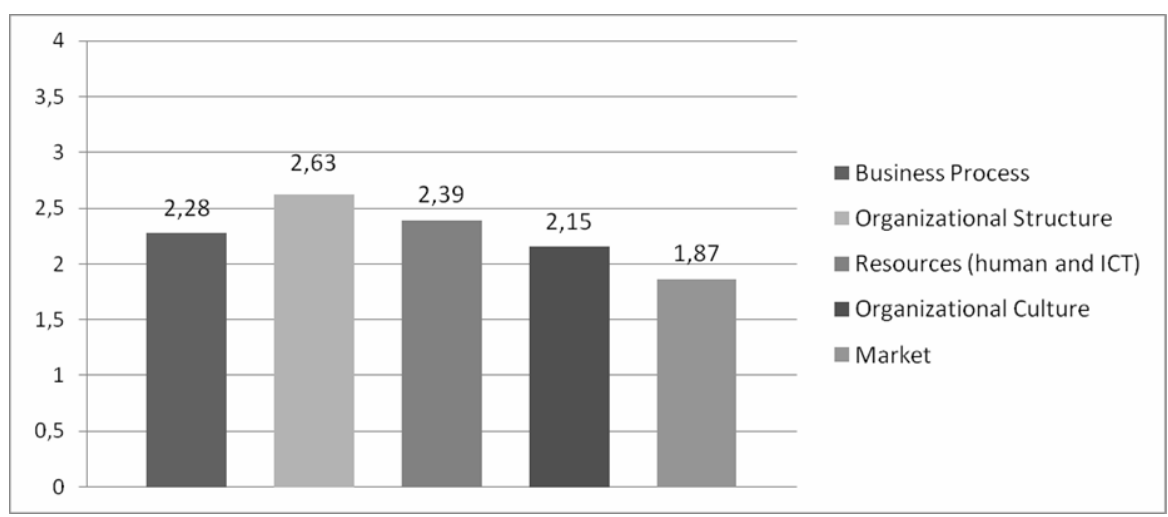

Fig. 3. Industries' Preparedness for each Analyzed Perspective

preparation, getting an average of 2.63. This result has been obtained because most of the industries are well-structured in departments physically separated, and their employees have well-defined functions. This characteristic is essential for industries that want to be part of a VBE because it supports their inter-organizational communication. Regarding the human and ICT resources it can be verified that the industries utilize them in a moderated way, reaching 2.39 as average. However, assessing each industry separately it could be verified that there is a considerable difference among them, where some of them are highly automated while others are poorly automated. This discrepancy impacts negatively in the VBE implementation process because everyone needs to have a minimal ICT bases to collaborate.

Considering the organizational culture, it could be observed in Figure 3 that most of the industries do not stimulate neither internal nor external collaboration. This low preparedness, with 2.15 of average, represents the biggest problem to implement the VBE for NuFerJ because to slight improve this perspective it is necessary a deep change in institutional and organizational principles, and this takes a long time to present results. About the market, this perspective has reached the lowest average among all the perspectives evaluated, which is 1.87 . This has been cased mainly because the industries are not habituated to make strategic planning. For instance, several industries do not have budget planning longer that one year. The industries should improve this aspect before applying to take of a VBE.

\section{Implementation Methodology Specification}

The last step of cluster mapping is to design the VBE implementation methodology. This methodology aims at identifying all the activities, and their sequence of execution, necessary to cope with the main aspects modeled in the VBE model. The activities presented in the methodology are specified based on the main issues stated in the NuFerJ VBE model [8] (see section 3). However, the activities positioning inside the methodology is guided by the preparedness assessment done previously and presented 
in section 5. The methodology specified in BPMN ${ }^{1}$ [12] identifies which steps should be done within each VBE life cycle phase [4]. Next paragraphs detail the proposed NuFerJ implementation methodology.

VBE Creation (Foundation): As showed in Figure 4, this phase defines the VBE mission and goals, its strategies in terms of marketing, political and economical issues, and its operating rules and duties. The VBE usually begins with strategic members selected by a sort of commission. In NuFerJ, these strategic members have been selected by means of a board composed of the NuFerJ managers and some invited industry managers. Business processes, ICT infrastructure and governance structure definitions complete the list of activities in this phase. Considering industries' market preparedness assessment done before, it can be noted that the definition of marketing and economical strategies is the bottleneck. Considering that these two activities are performed in parallel with other activities, it is important to put more attention and workforce in such activities in order to do not delay the whole methodology deployment. So, it would be suitable contract external marketing and financial consulting considering that the cluster does not present expertise about such subjects inside it.

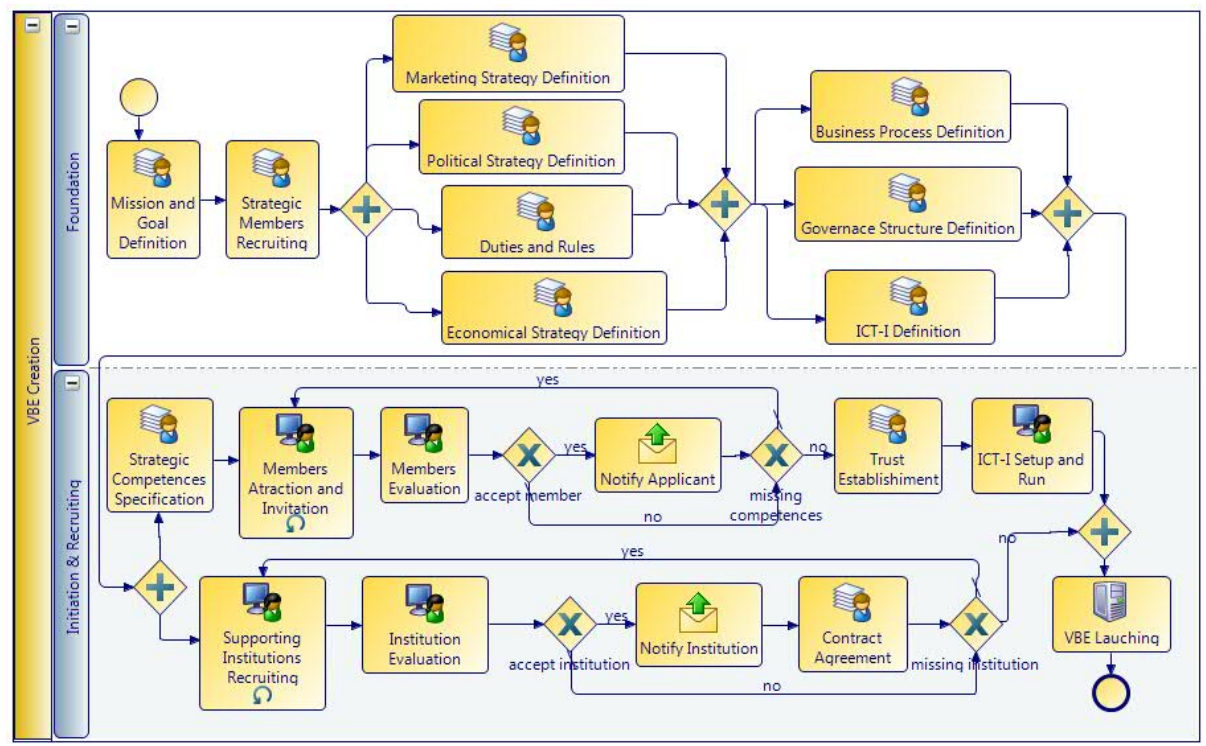

Fig. 4. NuFerJ Implementation Methodology - Creation phase

VBE Creation (Initiation \& Recruiting): As can be seen in Figure 4, this phase is concerned to recruiting the VBE members and the VBE supporting institutions (e.g. educational and financial institutions). Considering the VBE members, the envisaged competences should be firstly specified in order to invite the right members. Every candidate should be empowered with basis knowledge on how to work in a VBE, which includes the aspect of trust, governance, etc. Once a given industry is

\footnotetext{
${ }^{1}$ BPMN - Business Process Modeling Notation (www.bpmn.org).
} 
considered prepared to become a member, its ICT infrastructure is prepared. Having all this prepared, the VBE can be launched. Based on the organization culture preparedness it can be identified that the establishment of trust will take time. In fact, this is one of the main barriers when people or entities are collaborating. There are no standard strategies to establish trust. Therefore, in NuFerJ the strategy is to stimulate the good relationship among the preselected strategic members, and through punctual initiatives tries to aggregate more members in this reliable and trustworthy group.

VBE Operation (VBE management): Figure 5 shows that VBE management comprises the activities related to deploy VBE business processes and systems. In NuFerJ the processes (and further systems) of Membership and ICT infrastructure management should be introduced first. Profile and Competence Management comes after and includes the deployment of a system to register and maintain VBE members' information. After that, a number of activities can start in parallel: Financial, Marketing, Performance, Acquisition, Trust, VO inheritance and Customer Relationship management. Considering the resource assessment done previously, it could be observed that NuFerJ members have a high difference of ICT resource utilization. It implies that some of the involved industries need to allocate more efforts to reach the same level of preparedness presented by the other members (such preparedness is mandatory to deploy the aforementioned systems). This preparation can imply to spend money to buy new technologies. That is a critical issue when talking about SME and it can make some of the participants to give up the process. Besides that, acquisition management is critical for NuFerJ because it has a tremendous impact on the mould final cost, so it can represent to win or lose the business. Therefore, this is a concrete aspect where working collaboratively (i.e. buying together) can bring advantages.

VBE Operation (VO creation): Figure 5 shows that VO creation encompasses activities devoted to initiate new VEs once new collaboration opportunities are identified. Typically, Opportunity Identification management is deployed first. This seems natural as it is responsible for brokering business opportunities. Opportunity Quotation management is the next one. Based in the business process assessment, this is another critical task that must be paid attention because most of the quotation employees do not know how to exactly quote a mould. They used to do this either by feeling or based on quotations done by other industries in a round of quotation. Following the methodology, the next step is to deploy the partners selection management. For that, it is necessary first the deployment of performance and trust management. As already mentioned, trust is specially complicated when talking about collaboration. So, the deployment of trust management process and system will take time mainly due to the fact that it will be hard to reach a common agreement among the involved industries about how this process should be constituted and handled. The remaining activities are performed in sequence: Contract Negotiation management (among VO members, and between the VBE and the customer), VO Planning management (to specify which partners will do what and when), VO Registration (to prepare the VBE's repositories to store VO's information for further inheritance), and Launching management (to coordinate the VO starting). Considering this last set of activities, contract negotiation management has to be carefully performed because it includes not only technical, but also legal issues. So, as legislations and bylaws can 
change rapidly, this process and system should be flexible to quickly attend and assimilate such changes.

As can be seen in Figure 4 and Figure 5 the methodology has a suitable number of activities what implies that it would take long to be entirely deployed. However, meanwhile, some economical, legal or financial facts can change in the country, or even in the world, and these things could affect the normal VBE deployment. So, these changes should be rapidly assimilated by the methodology during its deployment process. Although not explicitly represented in the methodology, any activity can be replaned or changed, even if this implies replanning the remaining activities. Moreover, if an activity impacts backward in the execution flow, this feedback should be passed to the previous executed activities and, if necessary, these affected activities should be replanned and executed again.

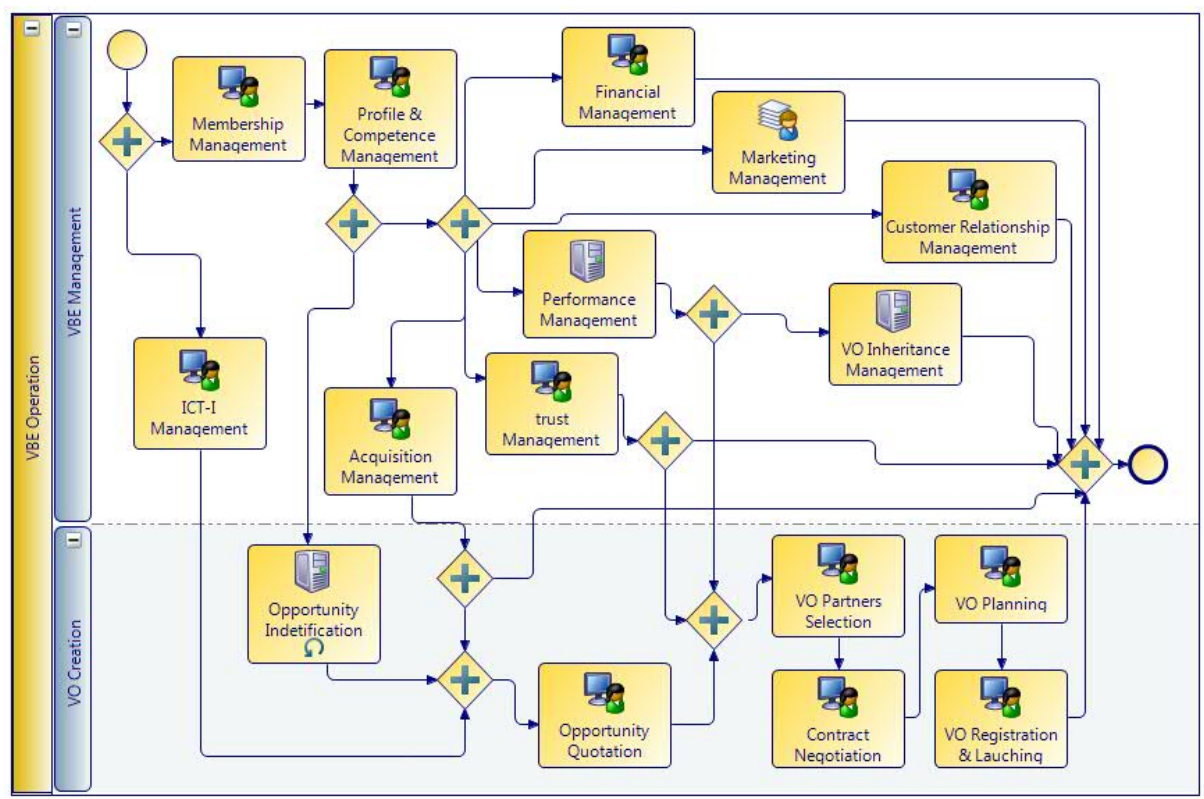

Fig. 5. NuFerJ Implementation Methodology - Operation phase

\section{Conclusions}

This paper presents a set of guidelines conceived to transform industry clusters in VBEs. Its main contribution is to systematize the whole process that concerns the implementation of a VBE when the organizations are previously arranged as a cluster. It also presents the first results of this ongoing research that is being applied in a case study for more than one year.

In this work it has been applied a top-down approach to derive comprehensive instances of VBEs based on CNOs reference models. This standardized support facilitates further VBE scalability and configurability because the VBE model is 
conceptually created considering most of the aspects related to a VBE, even if some of these aspects are not been considered in a first stage. The proposed set of guidelines have been devised mainly to supporting the implementation of VBEs from the mould and die sector. But, its natural evolution is to be extended and adapted to be used as guideline to transform in VBE any kind of cluster.

In fact, the aim of this set of guidelines is to support a better specification of the VBE implementation methodology. However, the deployment of this methodology depends on the particularities and preparedness level of the candidates to became VBE members. This means that the methodology's steps can present different levels of complexity and take different times to be implemented. In a first estimation, it is planned that the entire execution of the methodology with the creation of the first VO would take almost three years to be concluded.

Due to its practical conceptualization, even based on reference frameworks, there are no formal methods to evaluate the set of guidelines, especially the methodology, in order to assess its correctness and effectiveness. This assessment will be gradually done during its execution, not only in NuFerJ cluster, but also in many other study cases. So, only this real assessment can provide suitable feedback to ensure the guidelines correctness, or provide valuable information to fix and / or improve them.

Next steps of this work are mainly concerned to a deeper evaluation of the methodology considering a larger number of NuFerJ's members as well as applying this set of guidelines in other clusters that want to become a VBE or even creating a VBE from scratch (where its members do not belong to a previous established cluster). This last initiative should be carefully evaluated, because the set of guidelines have not been structured to create VBE from scratch, and thus it could not be prepared to perform such thing. However, its utilization can be tested and some adaptations may be proposed.

Acknowledgements. This work has been partially supported by Brazilian Council for Scientific and Technological Development - CNPq. The authors thank NuFerJ's managers, in particular Mr. Alexandre Wanzuita and Mr. Christian Dihlmann, as well as the industry managers that are participating and providing contribution for this work.

\section{References}

1. Porter, M.E., Fuller, M.B.: Coalitions and global strategy. Competition in Global Industries 1(10), 315-343 (1986)

2. Vallejos, R.V., Lima, C.P., Varvakis, G.: Towards the development of a framework to create a virtual organisation breeding environment in the mould and die sector. Journal of Intelligent Manufacturing 18(5), 587-597 (2007)

3. Camarinha-Matos, L.M.: Emerging Collaboration Forms And Further Research Needs. In: Methods and Tools for Collaborative Networked Organizations, pp. 513-528. Springer, New York (2008)

4. Afsarmanesh, H., Camarinha-Matos, L.M.: A Framework for Management of Virtual Organization Breeding Environments. In: Sixth IFIP Working Conference on Virtual Enterprises. Springer, Valencia (2005) 
5. Galeano, N., et al.: VBE Pilot Demonstrators. In: Camarinha-Matos, L.M., Afsarmanesh, H., Ollus, M. (eds.) Methods and Tools for Collaborative Networked Organizations, pp. 405-430. Springer, New York (2008)

6. Romero, D., et al.: Towards the Next Generation Collaborative Networked Organizations: International Challenges, Trends and Research Opportunities (2009), http: / / www . uninova.pt/ prove09/2009/presentations / PRO-VE09_Special_Panel_Session_ebook.pdf (cited 2010 03/10/2010)

7. Romero, D., Galeano, N., Molina, A.: A Virtual Breeding Environment Reference Model and its Instantiation Methodology. In: Ninth IFIP Working Conference on Virtual Enterprises. Springer, Poznan (2008)

8. Baldo, F., Rabelo, R.J.: For a Methodology to Implement Virtual Breeding Environments - A Case Study in the Mold and Die Sector in Brazil. In: Tenth IFIP Working Conference on Virtual Enterprises. Springer, Thessaloniki (2009)

9. Camarinha-Matos, L.M., Afsarmanesh, H.: The ARCON Modeling Framework. In: Camarinha-Matos, L.M., Afsarmanesh, H. (eds.) Collaborative Networks: Reference Modeling, pp. 67-82. Springer, New York (2008)

10. SOCIESC, Projeto Pesquisa de Mercado Interno para o APL Metalmecânico de Joinville. SOCIESC - Educational Society of Santa Catarina, Joinville, p. 57 (2007)

11. Camarinha-Matos, L.M., et al.: ARCON Reference Models for Collaborative Networks. In: Camarinha-Matos, L.M., Afsarmanesh, H. (eds.) Collaborative Networks: Reference Modeling, pp. 83-112. Springer, New York (2008)

12. White, S.: Introduction to BPMN. IBM Cooperation, p. 2008-029 (2004),

http: / / www. bptrends.com/publicationfiles/

07-04\%20WP 20 Intro\%20 to 20 BPMN $\% 20-\% 20$ White.pdf

(accessed in 02/03/2010) 